\title{
TOWARDS OPTIMIZATION OF BUILDING ENERGY AND OCCUPANT COMFORT USING MULTI-AGENT SIMULATION
}

\author{
Laura Klein ${ }^{1}$, Geoffrey Kavulya ${ }^{1}$, FarrokhJazizadeh ${ }^{1}$, Jun-young Kwak ${ }^{2}$, Burcin Becerik-Gerber ${ }^{1}$, \\ Pradeep Varakantham ${ }^{3}$ and MilindTambe ${ }^{2}$ \\ ${ }^{1}$ Department of Civil and Environmental Engineering, University of Southern California, Los Angeles, USA \\ ${ }^{2}$ Computer Science Department, University of Southern California, Los Angeles, USA \\ ${ }^{3}$ Singapore Management University, Singapore \\ *Corresponding author (becerik@usc.edu)
}

\begin{abstract}
The primary consumers of building energy are heating, cooling, ventilation, and lighting systems, which maintain occupant comfort, and electronics and appliances that enable occupant functionality. The optimization of building energy is therefore a complex problem highly dependent on unique building and environmental conditions as well as on time dependent operational factors. To provide computational support for this optimization, this paper presents and implements a multi-agent comfort and energy simulation (MACES) to model alternative management and control of building systems and occupants. Human and device agents are used to explore current trends in energy consumption and management of a university test bed building. Reactive and predictive control strategies are then imposed on device agents in an attempt to reduce building energy consumption while maintaining occupant comfort. Finally, occupant agents are motivated by simulation feedback to accept more energy conscious scheduling through multi-agent negotiations. Initial results of the MACES demonstrate potential energy savings of 17\% while maintaining a high level of occupant comfort. This work is intended to demonstrate a simulation tool, which is implementable in the actual test bed site and compatible with real-world input to instigate and motivate more energy conscious control and occupant behaviors.
\end{abstract}

Keywords: Building Energy Consumption, Multi-agent Systems, Occupant Comfort, Multi-objective Optimization

\section{INTRODUCTION}

Buildings in the United States account for $40 \%$ of national energy consumption of which $36 \%$ is consumed by heating, cooling, and ventilation systems, $18 \%$ is consumed by lighting systems, and $10 \%$ is consumed by computers and other appliances [1]. These systems and devices are essential for maintaining occupant comfort and enabling occupant functionality. Energy reduction strategies for building operations must therefore simultaneously address both energy system controls, executed by mechanical and electrical equipment, and energy system demands, imposed by building occupants. Today, building systems generally operate according to fixed schedules, maximum designoccupancy assumptions, and code defined occupant comfort ranges to ensure satisfactory temperatures, ventilation, and luminance at all times.Observations of actual building occupancy have found average occupancy in office buildings to represent at most a third of their design occupancy, even at peak times of day [2]. There is a great potential therefore to reduce building energy consumption by tailoring system control to actual occupancy loads. Even more, recent studies have shown weak and context dependent correlations between code- defined comfort ranges and occupant reported comfort ranges [3-5]. Often times, occupant comfort ranges are found to be larger and more forgiving than predictedranges implying a potential for reduced building energy consumption by allowing more flexible and adaptive control of heating, ventilating, and air conditioning (HVAC) and lighting system set points [6, 7]. To provide computational support for this optimization, this paper presents and implements a multi-agent comfort and energy simulation (MACES) to model alternative management and control of building systems and occupants. During the simulation, energy consumption is 
computed and analyzed at the zone level where the contributions of individual occupants and devices can be investigated and modified to reduce building energy consumption without compromising functionality and occupant comfort. Human and device agents are used to explore current trends in energy consumption and management of a university test bed building. Reactive and predictive control strategies are then imposed on device agents to accommodate actual occupancy and occupancy preferences. Occupant agents are also motivated bysimulation feedback to accept more energy conscious scheduling through multi-agent negotiations. The energy impacts of each control strategy are analyzed to understand where the most significant reductions are realized and where opportunities exist for future control strategies. Differentiating itself from other multi-agent building simulations, the MACES is intended to be compatible with real-world input so that it is implementable in the actual test bed site and can accurately represent occupant behavior including occupant preferences.

\section{MULTIAGENT SIMULATION SYSTEMS}

In computer science, agents, a type of distributed artificial intelligence, include physical or virtual entities that intelligently interact in an environment. Multi-agent frameworks are used to model complex environments with multiple cyber agents in simulations or physical agents that act in the world. Multi-agent simulations may serve several distinct functions including (1) evaluative, allowing policy makers and operators to understand how current systems work, (2) speculative, developing hypotheses for possible changes to the system, and (3) educational, informing the decisions by designers of future systems.Multiagentsystems have been employed in smart home management[9] and in building energy optimization and control [10]. These systems, however, deal only with device agent management and with energy optimization for unoccupied buildings and do not incorporate occupant preferences or decisions. [12, 13] proposed multi-agent building control systems that manage occupant comfort preferences as well as building energy. These systems, however, have only been evaluated through simulations of single zones that do not fully represent the complexities of an actual operational commercial building.

\section{SIMULATION TEST BED}

The developed MACES is tested and studied with a complete floor of a three-story university building. The test bed floor was selected as representative of a typical commercial facility including offices, classrooms, and conference rooms occupied by approximately 30 faculty and staff and 700 students. The floor is divided into 17 thermal zones and 33 rooms based on the actual zoning of Variable Air Volume (VAV) boxes in the HVAC system. Generally, a single thermal zone includes one classroom ortwo or three smaller offices. Lighting power loads are derived directly from building plans for all rooms. The floor and window areas for each room and zone are also extracted directly from building plans for thermal load calculations. Actual classroom schedules are used to model temporary(student) occupant andrepresentative meeting/work schedules are generated for permanent (faculty and staff) occupants. Actual outdoor temperatures recorded in Los Angeles, CA at the test bed site over a 24 hour period in January are used for energy simulations.

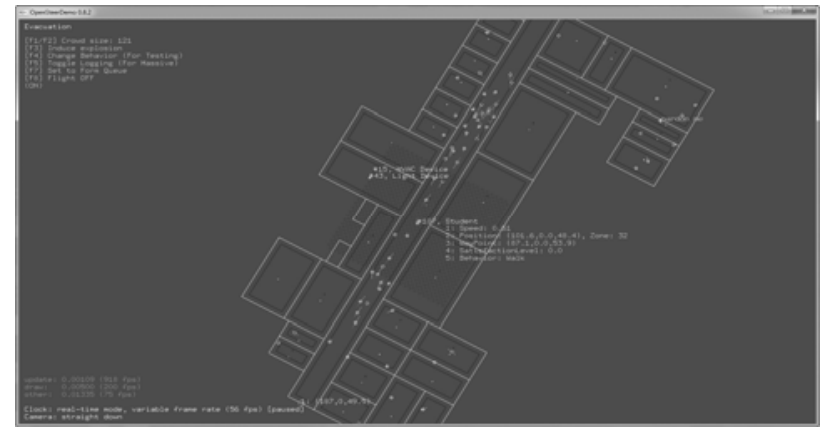

Fig. 1 Building floor plan modeled in MACES with thermal zoning and areas.

\section{SIMULATION AGENTS}

The MACES consists of a simulation module, an input/output module to communicate with agents, and an underlying reasoning and planning module. In particular, the input/output module first collects data and constructs the world model. Given the world model, the reasoning and planning module generates policies to achieve the given objectives in the context of coordination. With these world 
model and generated policies, the simulation module models agents' physical and behavioral interactions in the system and realizes the coordination in the actual world via the input/output module.

Agents modeled in the simulation consist of device and human agents. Device agents include HVAC agents, lighting agents, and appliance agents. The HVAC agents represent Air Handler Units (AHU), VAV boxes, and temperaturesensors that monitor and control the temperature and ventilation in their assigned zones by adjusting supply air temperatures and flow rates. The lighting agents control the lighting levels of their assigned rooms according to actual switch controls. Appliance agents represent computers including desktops and laptops and operate according to two states: "on" and "standby". To represent actual occupants of the test bed building, human agents include permanent occupants such as faculty and staff in offices and attending meetings, and temporary occupants such as students or faculty and attending classes. Each human agent can access a subset of the six available behaviors according to their types - wander, attend the class, go to the meeting, teach, study, and perform research, any one of which may be active at a given time, where the behavior is selected via the given class and meeting schedules. Each agent also has a stochastically determined temperature preference (normal distribution between $65^{\circ} \mathrm{F}$ and $80^{\circ} \mathrm{F}$ ).

\section{BUILDING SYSTEM CONTROL STRATEGIES}

The simulation uses agents to investigate the energy and occupant comfort consequences of four building control strategies related to HVAC, lighting, and appliance management. The first control strategy, Manual, represents the assumed baseline system where temperature is regulated according to two set ranges for occupied $(70 \circ \mathrm{F}$ $75 \circ \mathrm{F})$ and unoccupied periods $(50 \circ \mathrm{F}-90 \circ \mathrm{F})$ of the day and lighting is operated by humans. In particular, human agents always turn on the lights when they enter a room and stochastically turn off lights when they leave a room. Appliances are assumed to be always on. In the second control strategy, Reactive, HVAC, lighting, and appliance device agents respond to actual occupancy and occupant preferences detected in each zone throughout the day. In the third control strategy, Proactive, device agents adjust temperature and lighting according to scheduled occupancy and the known occupant preferences of the predicted occupants. Finally for Proactive-MDP, Markov Decision Problems (MDPs) are used to model and modify agent interactions,specificallyfocusingonrescheduling meetings. The Proactive-MDP control strategy uses predicted energy consumption and occupant preferences to develop and negotiate more energy and comfort conscious meeting alternatives for human agents. MDP allows the modeling of random outcomes associated with human behaviors such as the uncertainties of occupants accepting changes to their schedules.

\section{ENERGY AND COMFORT}

The energy and comfort outcomes of each control strategy are estimated in real-time throughout the simulation as well as predicted to aid in MDP optimization. The multiobjective optimization of building energy and occupant comfort requires real-time cost functions to be used to represent room, zone, and occupant level values for these parameters. Energy consumption for lighting and appliance agents are modeled according to each agent's assumed power and their current state: "on", "off”, or "standby" as shown in Table 1. Lighting, appliance, and human agents are also assumed to generate the heat loads shown in Table 1 [12]. Additionally, heat transmission and solar gains through windows in perimeter zones areestimated according to the test bed building location, outdoor temperatures, time of day, and glazing areas.

Table 1 Lighting and appliance power loads (W)

\begin{tabular}{l|lccc}
\hline Agent Type & Category & On & Off/Standby & $Q$ \\
\hline \multirow{2}{*}{ Lighting } & Office & 128 & 0 & 128 \\
& Conference & 192 & 0 & 192 \\
& Classroom & 768 & 0 & 768 \\
\hline \multirow{2}{*}{ Appliance } & Desktop & 150 & 10 & 150 \\
& Laptop & 50 & 5 & 50 \\
\hline Human & - & - & & 75 \\
\hline
\end{tabular}

HVAC energy consumption is modeled by real-time 
estimation of each zone's total thermal heat load $\left(Q_{z}\right)$. Real-time occupancy is used to calculate minimum outdoor ventilation $\left(\mathrm{V}_{\mathrm{O}}\right)$ rates according to ASHRAE 62.1. The temperature of the mixed air $\left(\mathrm{T}_{\mathrm{MA}}\right)$ entering the $\mathrm{AHU}$ is computed as a linear function of the ratio and temperature of outside air and the ratio and temperature of the current zone temperatures. The supply air temperature $\left(\mathrm{T}_{\mathrm{S}}\right)$ is set to55 $\mathrm{F}$ and the required supply air volume $\left(\mathrm{V}_{\mathrm{S}}\right)$ is calculatedto offset $\mathrm{Q}_{z}$ and to achieve the desired change in temperature over time $(\Delta T)$ from the current zone temperature $\left(\mathrm{T}_{\mathrm{Z}}\right)$ according to the zone volume $\left(\mathrm{V}_{\mathrm{Z}}\right)$.

\section{$V s=[(1.1 \times \operatorname{Vz} \times \Delta T)+Q z] /[1.1 \times(T z-T s)](C F M)$}

Total HVAC energy consumption $\left(\mathrm{Q}_{\text {TOTAL }}\right)$ is calculated as the sum of the following two equations for fan distribution energy (QFAN) and chilled water cooling energy (QCHILL) where 1.25 and 0.06 representperformance coefficients of the actual test bed fans and chilled water production plant.

$\mathrm{Q}_{\mathrm{FAN}}=1.25 \times \mathrm{V}_{\mathrm{S}}$

$Q_{\text {CHILL }}=0.06 \times 1.1 \times\left(T_{M A}-T_{S}\right) \times V_{S}$

Average occupant comfort is estimated using the Predicted Mean Vote (PMV) thermal sensation index which calculates the average occupant sensory perception on a 7point scale ranging from cold to hot, according to input of the thermal variables listed in Table 2 [13]. All thermal variables are kept at the constant values below with the exception of temperature, which is controlled throughout the simulation.

Table 2 Fixed parameter values for PMV calculations

\begin{tabular}{|l|l|l|l|}
\hline Clothing (Clo) & 1 & Air Velocity & $0.1 \mathrm{~m} / \mathrm{s}$ \\
Metabolic Rate & 1.2 & Relative Humidity & $40 \%$ \\
External Work & 0 & Temperature & Varies \\
\hline
\end{tabular}

\section{PRELIMINARY RESULTS}

Running the developed MACES over a 24 hour period demonstrated reductions in energy consumption for all three of the alternative control strategies in comparison to the baseline control system. Proactive and ProactiveMDP saw the most significant energy savings with $15.1 \%$ and $17.4 \%$ energy reductions respectively. Reactive control realized an energy savings of $11.8 \%$ as compared to the baseline system. The most significant reductions in energy consumption were achieved during the assumed occupied time of day when occupant behaviors could be modified and occupant preferences could be used to optimize set points. During occupancy, the impact of MDP rescheduling meeting alternatives could also be realized. As shown in Figure 2, while Reactive control saw the least energy savings, it saw the greatest energy reduction during a single half-hour time step. Throughout the simulation, Proactive and Proactive-MDP showed almost identical energy consumption with Proactive-MDP usually consuming slightly more energy with the exception of hours 2-3:30. During this time, Proactive-MDP allowed an approximate $8 \%$ savings over Proactive control indicating successful rescheduling of meetings for energy optimization.

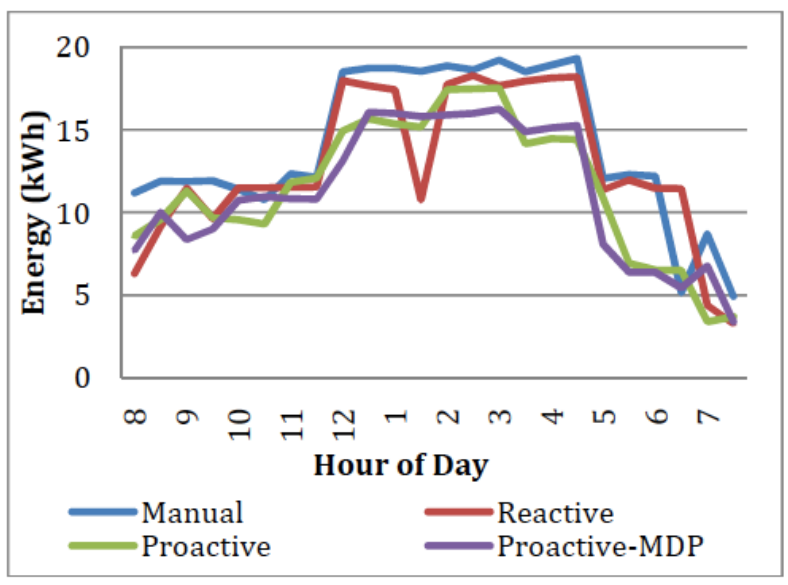

Figure 2. Building energy consumption over 12 hour occupied period for four control strategies.

Investigating the impact of the Proactive-MDP further, energy consumption was broken down to zone level consumption for the simulated day. As shown in Figure 3, Proactive-MDP reduced energy consumption in only three of seventeen HVAC zones (in zones 13-15), and only significantly reduced energy consumption in two of these zones. Zones 13, 14, and 15 represent five conference rooms in the test bed building and therefore are highly subject to energy reductions by meeting rescheduling. When the number of meeting participants allowed, the MDP model was able to suggest rescheduling of meetings in these conference rooms to smaller offices where HVAC and lighting demands would be significantly less to heat, 
cool, and/or illuminate the meeting space to desired levels. This rescheduling of meetings to smaller office spaces is demonstrated by the slight increases in energy consumption in most of the other zones.

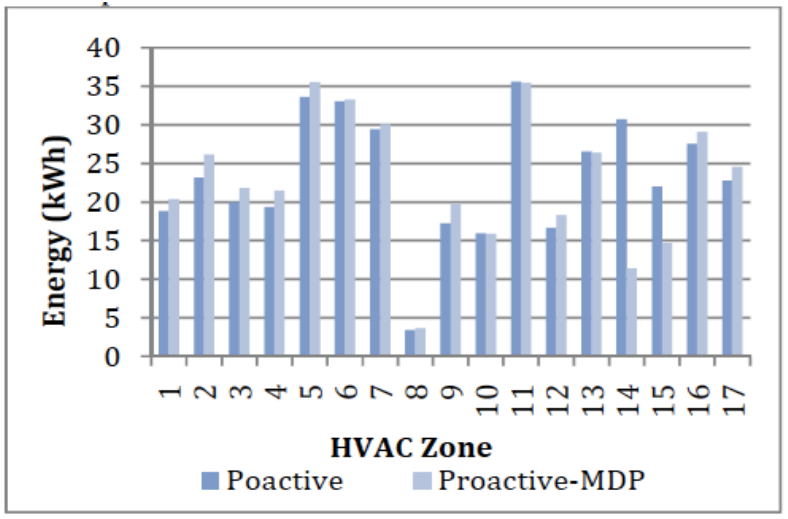

Figure 3. Energy consumption by zone for Proactive and Proactive-MDP control strategies.

Zone 11 had the greatest energy consumption for both control strategies reflecting the fact that this zone includes three large offices with large windows on the south side of the building. As solar gains are significant contributors to zone heat gain and HVAC cooling demands, MDP control should be further refined to negotiate more rescheduling out of this zone to interior office or conference zones in future simulations. Zones 5, 6, and 7 represent classrooms and the next greatest consumers of energy after Zone 11 . Rescheduling of classes with the MDP was not attempted as classes are generally scheduled on a permanent basis. Future simulation runs, however, could be informative for classroom scheduling, taking advantage of times of day for reduced solar gain and consequential heating, cooling, and/or lighting. Implementing MACES for buildings with significantly more classrooms and fewer offices could also allow for energy optimization of classrooms based on classroom size and predicted occupancy as demonstrated with offices in the test bed building.

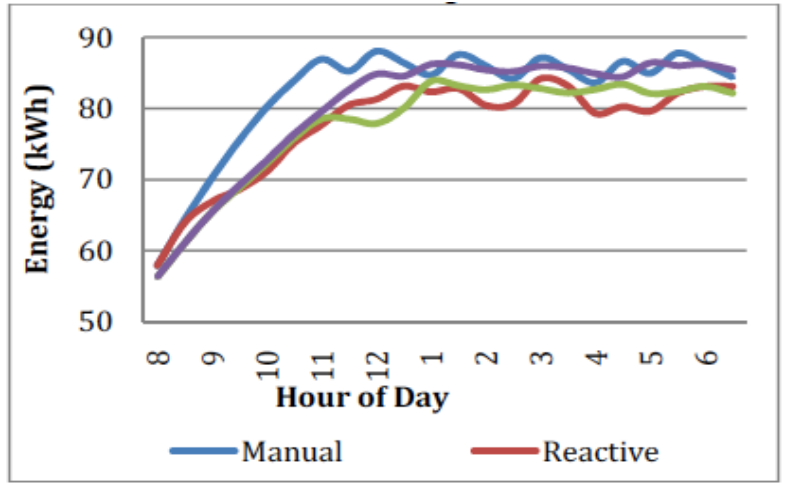

Figure 4. Percent of satisfied occupants during occupied period for four control strategies.

Inadditionto reducingenergy consumption,thethree alternativecontrolstrategiesalsosuccessfully managed occupantcomfort.ASHRAE 55-2004,requires thermal indoorconditionstosatisfy atleast $80 \%$ of occupants.As shownin Figure4,thislevelwas achieved and approximatelymaintainedby allsimulationcontrol strategies.The delay in occupantsatisfaction reaching this levelatthebeginning of thesimulation resultedfrom the factthat all interiorspacesstartedat $50 \circ \mathrm{F}$ at hour8. Accordingtothesimulation,Manualcontrolmaintained the highest level of occupant satisfaction, with approximately $85 \%$ of occupants satisfied throughout the day. This satisfaction level, however, was closely matched by the Proactive-MDP control which showed a noticeable improvement in comfort compared to Proactive control without MDP. This improvement with MDP implementation demonstrates the impact of negotiating rescheduling to optimize not only energy but also to accommodate occupant preferences. MDP optimization of comfort was achieved by negotiating back-to-back meetings that ensured desirable temperature conditions upon arrival of meeting participants with minimal workrequired of HVAC agents. Reactive control resulted in the lowest and most variable satisfaction levels. Such variance and dissatisfaction can be explained by the fact that with Reactive control, temperatures were adjusting according to occupant preferences only after they entered a zone causing a time delay between occupancy and achieved comfort levels. 


\section{CONCLUSIONS \& FUTURE WORK}

The preliminary results of the MACES demonstrate promising improvements to both building energy and occupant comfort management. The implementation of the Proactive-MDP control strategy especially optimized both energy demands and occupant preferences resulting in over a $17 \%$ reduction in energy from the baseline, and approximately $85 \%$ occupant satisfaction. The majority of energy savings achieved by MDP negotiations were realized in two of the seventeen HVAC energy zones. This finding indicates potentially further energy reductions possible by using MDP to reschedule out of southern perimeter zones and to negotiate classroom schedules. This work shows successful implementation of the developed MACES on a real-world, multi-zone test bed building involving complexities of multiple human and device agent types. Further research will include incorporation of actual occupant preferences and deploying the simulation and MDP meeting agents to the actual test bed site. In this way, the simulation will be used to instigate and motivate more energy conscious occupant behaviors and to provide real world validation of the simulated energy savings.

\section{ACHNOWLEGEMENTS}

Authors would like to acknowledge the Department of Energy's support of this project through funding for the Building Level Energy Management (BLEMS) development project conducted at the University of Southern California (DOE Award DE-EE0004019). Any opinions, findings, conclusions, or recommendations presented in this paper are those of the authors and do not necessarily reflect the views of the Department of Energy.

\section{REFERENCES}

[1] Dep. of Energy, "Buildings Energy Data Book", 2009.

[2] Brandemuehl, M.J. and Braun, J.E., "Impact of demand-controlled and economizer ventilation strategies on energy use in buildings", ASHRAE, 1999.

[3] Barlow, S. and Fiala, D., "Occupant comfort in UK offices-How adaptive comfort theories might influence future low energy office refurbishment strategies", Energy Build., Vol. 39(7), pp.837-46, 2007.

[4] Corgnati, S.P., Filippi, M. and Viazzo, S., "Perceptionof the thermal environment in high school and university classrooms: Subjective preferences and thermal comfort", Build.Environ., Vol. 42(2), pp.951-9, 2007.

[5] Wagner, A., Gossauer, E., Moosmann, C., Gropp, T. and Leonhart, R., "Thermal comfort and workplace occupant satisfaction-Results of field studies in German low energy office buildings", Energy Build., Vol.39(7), pp.758-69,2007.

[6] Hwang, R., Cheng, M., Lin, T. and Ho, M., "Thermal perceptions, general adaptation methods and occupant's idea about the trade-off between thermal comfort and energy saving in hot-humid regions", Build.Environ., Vol.44(6), pp.1128-34, 2009.

[7] Nicol, J.F. and Humphreys, M.A., "New standards for comfort and energy use in buildings", Building Research and Information, Vol. 37(1), pp.68-73, 2009.

[8] Cook, D.J., Youngblood, M. and Das, S.K., "A multiagent approach to controlling a smart environment", Designing Smart Homes, pp.165-82, 2006.

[9] Lin, Z., Guiqing, Z., Bin, S., Xiuying, X. and Qiao, Y., "Building energy saving design based on multi-agent system", ICIEA, pp.840-4, 2010.

[10] Dounis, A.I. and Caraiscos, C., "Advanced control systems engineering for energy and comfort management in a building environment-A review", Renewable and Sustainable Energy Reviews, Vol.13(6-7), pp.124661,2009 .

[11] Qiao, B., Liu, K. and Guy, C., "A multi-agent system for building control", ICIAT, pp.644-50, 2006.

[12] ASHRAE, "Handbook: Fundamentals", 2009.

[13] Fanger, P.O., "Thermal Comfort", 1982. 